\title{
Universal Topological Properties of Two-Dimensional Trivalent Cellular Patterns
}

\author{
K. Y. Szeto, ${ }^{1}$ Xiujun Fu, ${ }^{1,2}$ and W. Y. Tam ${ }^{1}$ \\ ${ }^{1}$ Department of Physics, The Hong Kong University of Science and Technology, \\ Clear Water Bay, Kowloon, Hong Kong, China \\ ${ }^{2}$ Department of Physics, South China University of Technology, Guangzhou 510641, China
}

(Received 13 November 2001; published 19 March 2002)

\begin{abstract}
Universal topological properties of two-dimensional trivalent cellular patterns are found from shell analysis of soap froth and computer-generated Voronoi diagrams. We introduce a cluster analysis based on the shell model and find the universal relation $\ln \left(a / \mu_{2}\right)=A+B \ln \left(\mu_{2}\right)$, with the generalized Aboav parameter $a$ and second moment of the number of cell edge distribution $\mu_{2}$. For the second, third, and fourth shells of the cluster, $A$ and $B$ are the same for all samples. Furthermore, $A$ is increasing with shell number while $B$ is a universal number, -0.90 . For the first shell, the slope $B$ is the same for soap froths, but slightly different from Voronoi graphs.
\end{abstract}

DOI: $10.1103 /$ PhysRevLett.88.138302

Two-dimensional cellular structure constitutes a large class of patterns with important technological and scientific applications. Soap froth, polycrystalline grain mosaics, and biological tissues are natural examples of random, space-filling cellular networks [1]. Since cellular structures exist on scales ranging from microscopic to geological, much work has been devoted to the search for universal geometrical characteristics. Despite the enormous difference in length scales and different physical forces driving the evolution of the networks, there exist certain universal topological laws governing their similarity. These laws leave aside metrical properties (e.g., sizes of cells) and address the probability distribution $P_{n}$ of the number $n$ of edges of a given cell, or correlations between the numbers of edges of adjacent cells. One of the best-obeyed empirical laws is the Aboav-Weaire law [2,3] which states that on average the sum $[M(n) n]$ of the number of sides of the cells immediately adjacent to an $n$-sided cell is linear in $n$ :

$$
M(n) n \approx(6-a) n+\left(6 a+\mu_{2}\right),
$$

with the second moment $\mu_{2}=\sum_{n=3}^{\infty} P_{n}(n-6)^{2}$ being commonly used as a measure of the disorder. The notation $M(n)$ denotes the average number of edges of the adjacent cells to a given $n$-sided cell. The Aboav parameter $a$ is a measure of nearest neighbor correlation and for soap froth $a$ is approximately 1 .

There have been several important works published after the recognition of Aboav-Weaire law. Godreche et al. [4] have used planar graph theory in the context of counting planar Feynman diagrams with a cubic interaction to give an analytic expression of the Aboav-Weaire law in addition to the calculation of $P_{n}$. However, these exact results are based on a particular model and cannot explain the vast diversity of natural cellular patterns with different Aboav parameters and second moments. Delannay and Le Caer $[5,6]$ have studied the stationary topological properties of 2D cellular structures generated by random fragmentation in computer simulations, with very different $a$ and $\mu_{2}$ that falls on a universal curve that is valid for many systems,
PACS numbers: 82.70.Rr, 02.50.-r, 05.70.Ln

both natural and computer generated [5-8]. There are also many papers published on the two-cell correlation using maximum-entropy argument [9], but none of these theoretical efforts succeed in explaining the universality shown in the curve of $a / \mu_{2}$ vs $\mu_{2}$ (Fig. 2 of Ref. [5]).

We have studied soap froth as a typical two-dimensional cellular system under many different conditions such as temperature ramping [10], special sample preparation for particular initial configurations such as hexagonal bubble crystals [11], and evolution of the patterns under constant temperature environment [12-15]. These experimental studies have been supplemented by detailed theoretical analysis using the shell model [16-19] and numerical simulations $[20,21]$. The structural characterization of the soap froth in the shell model can be applied to all twodimensional trivalent cellular patterns. In soap froth we have shown certain universal features such as the generalized Aboav-Wearie law [16] for the average number $M(i, n)$ of edges of cells in the $i$ th shell of the central cell with $n$ sides. We also found that the number of cells $K(i, n)$ in the $i$ th shell is linear in $i$ with slope related to the Aboav parameter [17]. In this Letter, we study the generalized Aboav-Weaire law, which relates the correlation between the $i$ th shell neighbors with the disorder of the $i$-shell perimeter. We find new universal features for shells beyond the first.

Inspired by ideas from the renormalization group, we consider the generalization of the Aboav-Weaire law based on the shell model analysis $[17,18]$. We propose a generalized Aboav-Weaire law in the form

$$
M(N) N \approx\left(\langle N\rangle-a_{i}\right) N+b_{i} .
$$

Here $N$ is the number of inner perimeter edges in the $i$ th shell, $\langle N\rangle$ is the average value of inner perimeter edges of all the $i$ th shell clusters, and $M(N)$ is the mean number of edges of the cells in the $i$ th shell with inner perimeter edges $N$. This proposed equation is found to be obeyed by all the systems that we have analyzed, i.e., soap froths and Voronoi patterns from a random set of points. However, 
for different systems, the Aboav parameters $a_{i}$ and $b_{i}$ are different, and so is the second moment $\mu_{2}=\left\langle N^{2}\right\rangle-\langle N\rangle^{2}$.

In using the shell model, we must systematically handle the defects. In Fig. 1, we illustrate shells around a central bubble in an experimental soap froth pattern. Starting from a central bubble with $n$ edges, the first shell consists of all its $n$ neighboring bubbles. In Fig. 1, the central bubble is labeled 0 , and it is a hexagon. There is no defect in the first shell. After the first shell, where bubbles are labeled 1 in Fig. 1, we define the second shell as the set of bubbles which have at least one edge being the outer perimeter formed from the bubbles in the first shell. The bubbles in the second shell are labeled by 2 in Fig. 1 . We then define the outer perimeter of the second shell as the perimeter formed by those edges which meet new bubbles that are not labeled 0 or 1 . Now, recursively, we can define the third shell as the set of bubbles that are not labeled by 0 , 1 , or 2, and that share the edges in the outer perimeter of the second shell. We see in Fig. 1 that there are defects, which are bubbles with bracketed labels. Nondefective bubbles in the $i$ th shell are those that have neighbors in the neighboring shells, such as bubbles in the $i+1$ shell and the $i-1$ shell. However, a defect in the $i$ th shell does not have this feature, as can be seen in Fig. 1 for those bubbles with a bracketed shell number.

We have reported the details of the experimental works elsewhere [10,11]. Here we summarize our data on real soap froth for two samples, called $S 1$ and $S 2$. Our experiment was performed in a $26.7 \times 36.8 \mathrm{~cm}$ chamber consisting of two $1.6 \mathrm{~cm}$ thick rectangular Plexiglas plates separated by a $0.16 \mathrm{~cm}$ thick spacer. More than 20000 bubbles of $1 \mathrm{~mm}$ size were pumped into the chamber ini-

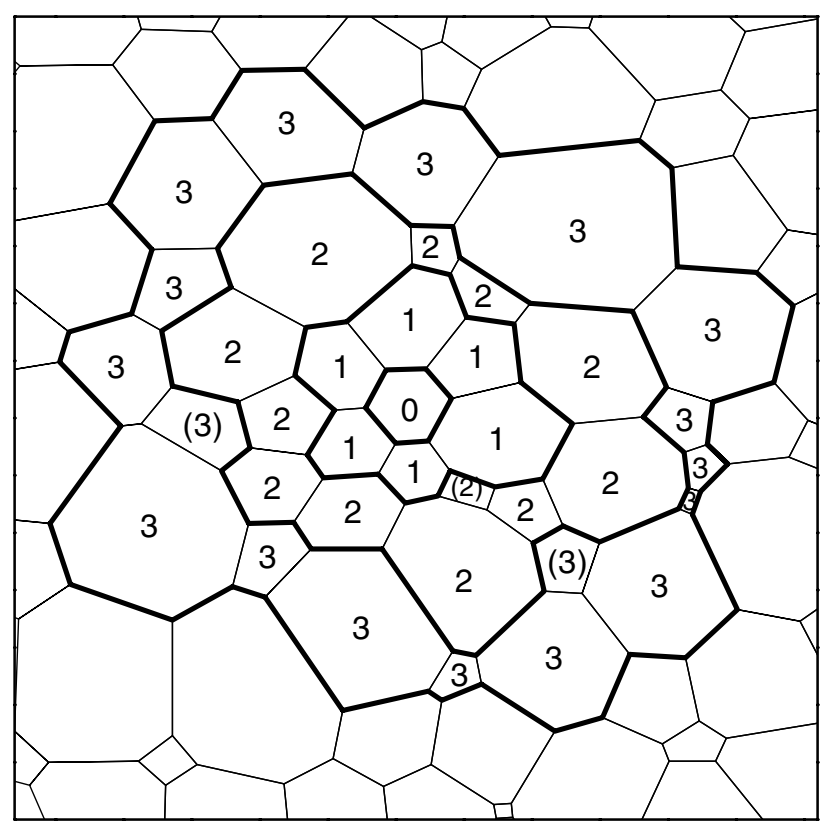

FIG. 1. Experimental soap froth data showing the shell structure in two-dimensional trivalent cellular pattern defined around a central cell, which is a hexagon labeled by 0 . The thick lines indicate the perimeters of the shells. Cells with bracketed numbers are defects.

tially so as to create a froth sample. Sample set $S 1$ refers to the set generated by an initially disordered state, while sample set $S 2$ refers to the ordered state which we called the bubble crystal [11]. The initial froth was drained to remove excess fluid. For the sample set $S 2$, defects were produced during the drainage, which led to the coarsening

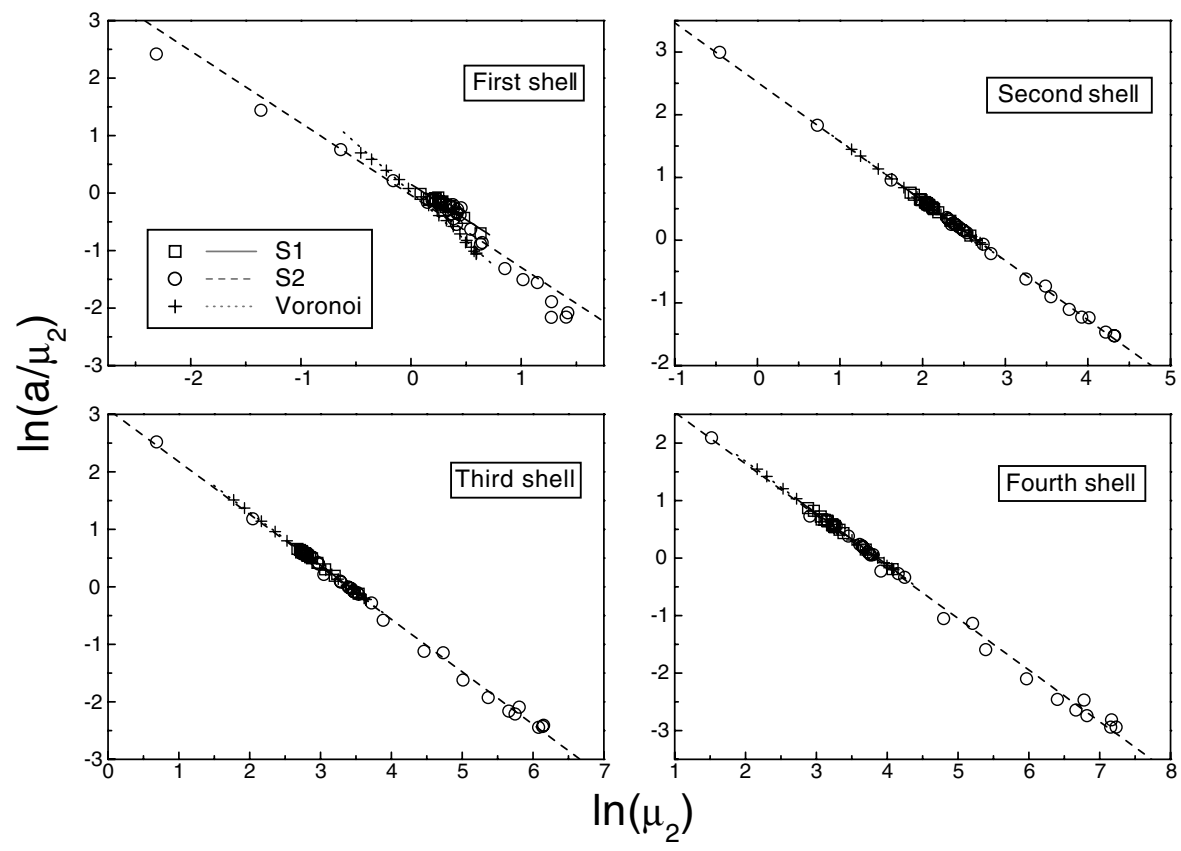

FIG. 2. Data collapse of $\ln \left(a / \mu_{2}\right)$ vs $\ln \left(\mu_{2}\right)$ for two soap froth samples $S 1$ (squares) and $S 2$ (circles) and the Voronoi networks (crosses). The solid, dashed, and dotted lines indicate the linear fits for the three sets of data, respectively. 
TABLE I. Results of the linear fit: $\ln \left(a / \mu_{2}\right)=A+B \ln \left(\mu_{2}\right)$.

\begin{tabular}{lccccc}
\hline \hline Shell & Sample & $A$ & Error $(A)$ & $B$ & Error $(B)$ \\
\hline \multirow{4}{*}{ First } & $S 1$ & +0.15 & 0.03 & -1.20 & 10.1 \\
& $S 2$ & -0.04 & 0.05 & -1.25 & 0.06 \\
& Voronoi & +0.01 & 0.01 & -1.70 & 0.04 \\
\multirow{5}{*}{ Second } & $S 1$ & +2.53 & 0.01 & -0.950 & 0.007 \\
& $S 2$ & +2.52 & 0.02 & -0.947 & 0.006 \\
& Voronoi & +2.53 & 0.002 & -0.955 & 0.001 \\
\multirow{5}{*}{ Third } & $S 1$ & +3.11 & 0.03 & -0.912 & 0.01 \\
& $S 2$ & +3.09 & 0.05 & -0.913 & 0.01 \\
& Voronoi & +3.15 & 0.006 & -0.926 & 0.002 \\
\multirow{5}{*}{ Fourth } & $S 1$ & +3.46 & 0.06 & -0.894 & 0.01 \\
& $S 2$ & +3.43 & 0.06 & -0.896 & 0.01 \\
& Voronoi & +3.49 & 0.01 & -0.905 & 0.003 \\
\hline \hline
\end{tabular}

of the froth. A high resolution charge-coupled device camera $(1037 \times 1344$ pixels $)$ was used to capture images of the froth every 10 min during its evolution.

The theoretical samples that we have investigated are Voronoi patterns constructed from sets of points randomly distributed in the plane. Different control levels on the minimal distance between any two points are used to generate different Voronoi cellular patterns. On the unit square, $N$ points are distributed randomly subjected to the constraint that distance between any pair of points is greater than $d$, with $0 \leq d<d_{\max }=1 / \sqrt{N}$. We sequentially place a point randomly on the unit square, and calculate the minimum distance $d_{i}$ between this point $i$ and all other existing points on the square. If $d_{i}>d$, then we accept this point, otherwise we reject it and generate a new point randomly. This process continues till we get $N$ points on the square. We have generated 17 random point sets with $N=10000$ and $d$ from 0 to $0.8 d_{\max }$ in steps of $0.05 d_{\max }$. The Voronoi diagrams are then constructed based on these 17 point sets. The cells in the Voronoi diagrams used in the actual analysis are about 9600, as we exclude the cells in the boundary.

We find a universal relation between $\mu_{2}$ and $a / \mu_{2}$ in each sample for analysis on the second, third, and fourth shells. This is shown in Fig. 2, where we have plotted the data for each shell, and all three samples produce data collapse on a straight line. The intercept $A$ and slope $B$ of the straight line are shown in Table I. From this table we see that the data collapse for the first shell is not very good, as can be seen in the difference in the value of the slope $B$. However, for the second, third, and fourth shells there is a universal relation for all samples and all shells that the value of the slope $B$ is -0.9 , while the intercept $A$ is shell dependent, but sample independent. Though both the intercept $A$ and slope $B$ are increasing with shell number, the difference between soap and Voronoi is negligible, and the dependence of $B$ on shell is small. The explanation of the topological properties of the two-dimensional trivalent pattern should thus be mainly mathematical in nature, though a small deviation in the first shell should be physical in origin. Effects due to a different physical or mathematical process generating the cellular pattern are very local, as the universality on the generalized Aboav parameters and fluctuation of the perimeters of giant clusters always prevails in higher shells. As our experimental data are limited, the statistics for shells higher than the fourth became poor, and we have not included it in our analysis. Nevertheless, we conjecture that the same universal properties hold for higher shells.

K. Y.S. acknowledges the support of RGC Grant No. HKUST6123/98P.

[1] J. Stavans, Rep. Prog. Mod. Phys. 56, 733 (1993).

[2] D. A. Aboav, Metallography 3, 383 (1974); 13, 43 (1980).

[3] D. Weaire, Metallography 7, 157 (1974).

[4] C. Godreche, I. Kostov, and I. Yekutieli, Phys. Rev. Lett. 69, 2674 (1992).

[5] R. Delannay and G. Le Caer, Phys. Rev. Lett. 73, 1553 (1994).

[6] G. Le Caer and R. Delannay, J. Phys. A Math. Gen. 26, 3931 (1993).

[7] N. Rivier, in From Statistical Mechanics to Statistical Inference and Back, edited by J. P. Nadal and P. Grassberger (Kluwer, New York, 1995).

[8] J. Lemaitre, A. Gervois, D. Bideau, J. P. Troadec, and M. Ammi, C. R. Acad. Sci. Paris 315, 35 (1992).

[9] M. A. Peshkin, K. J. Strandburg, and N. Rivier, Phys. Rev. Lett. 67, 1803 (1991).

[10] W. Y. Tam and K. Y. Szeto, Phys. Rev. E 53, 877 (1996).

[11] K. Y. Szeto and W. Y. Tam, in Stochastic Dynamics and Pattern Formation in Biological and Complex Systems, edited by S. Kim, K. Lee, and W. Sung (American Institute of Physics, New York, 1999), pp. 291-301.

[12] W. Y. Tam, R. Zeitek, K. Y. Szeto, and J. Stavans, Phys. Rev. Lett. 78, 1588 (1997).

[13] K. Y. Szeto and W. Y. Tam, Physica (Amsterdam) 221A, 256 (1995).

[14] K. Y. Szeto and W. Y. Tam, Physica (Amsterdam) 254A, 248 (1998).

[15] W. Y. Tam, K. M. Cheung, and K. Y. Szeto, Phys. Rev. E 57, 7354 (1998).

[16] K. Y. Szeto and W. Y. Tam, Phys. Rev. E 53, 4213 (1996).

[17] T. Aste, K. Y. Szeto, and W. Y. Tam, Phys. Rev. E 54, 5482 (1996).

[18] K. Y. Szeto, in Current Topics in Physics, edited by Y. M. Cho, J. B. Hong, and C. N. Yang (World Scientific, Singapore, 1998), Vol. 1, pp. 361-375.

[19] K. Y. Szeto, T. Aste, and W. Y. Tam, Phys. Rev. E 58, 2656 (1998).

[20] J. J. Chae Ph.D. thesis, University of Arizona, 1995.

[21] D. Brunett, J. J. Chae, W. Y. Tam, R. M. C. de Almeida, and M. Tabor, Phys. Rev. E 51, 5788 (1995). 